\title{
Reform and Practice of Deepening Diagnostics Teaching in Internal Medicine Internship
}

\author{
Shougang Wang \\ Beihua university affiliated hospital (First Clinic Medical College), Jilin, China \\ 704572350@qq.com
}

Keywords: Internal Medicine Internship, Deepening, Diagnostics Teaching

\begin{abstract}
Internal medicine covers a wide range of care types and is closely related to a variety of basic and clinical disciplines. The teaching of diagnostics should be deepened in internal medicine internship, and skill trainings should be carried out through clinical practices. By doing so, it will be made possible to broaden the interns' vision, enrich their clinical experience, and pave a better road for their future learning and professional career.
\end{abstract}

\section{Introduction}

Internal medicine covers a wide range of care types and is closely related to a variety of basic and clinical disciplines. It is considered a discipline that focuses the most on practicality in clinical teaching. How to deepen the content of diagnostics teaching throughout internal medicine internship has always been a topic pondered and explored by the clinical faculty for many years. However, most medical colleges emphasize the requirements on writing medical documents and conducting physical examinations, while ignoring the practical skills, such as the "techniques of punctures and incision", experimental diagnosis, imaging diagnosis, and electrocardiography. The lack of these types of knowledge makes it difficult for the interns to be handy and independent with the clinical diagnosis once they finish the residency. In particular, at the end of the internship, many interns could not even master the basic methods of ECG tracing. To solve this problem, based on the learning outcomes from the internship of our graduates in different teaching hospitals, reforms and practices have been proposed to deepen diagnostics teaching in internal medicine internship through comprehensive surveys and novelty assessment. After more than two years of clinical practice, it has been appreciated by the interns and achieved the desired results.

\section{Analysis of Status Quo}

In years of teaching and mentoring, we found that the graduates might have few hands-on opportunities due to various factors, such as the influences of medical setting, requirements for doctor's practice, self-guarding of patients, and the graduates' lack of theoretical knowledge or learning motivation. As a result, some interns may be unfamiliar or incompetent with the fundamental clinical operations, and, in particular, many interns could not even master the basic methods of ECG tracing at the end of the internship.

\section{Survey and Study}

We carried out intern workshops, mentor seminars, and questionnaires to evaluate the interns' overall performance, including both theoretical knowledge and basic operations, during the internship. After collecting feedbacks from all parties, timely summaries and adjustments were made to lay the foundation for developing specific requirements and training goals that are suitable for the interns. 


\section{Specific Measures}

\subsection{Selection of the control group.}

The intern team of Affiliated Hospital of Beihua University was selected as the internship group, and that of Jilin Petrochemical General Hospital was selected as the control group.

\subsection{Development of a proper and feasible internship plan.}

At the department of clinical internal medicine, most cases are the common and frequently-occurring diseases in the circulatory, respiratory, and digestive system. In this regard, two thirds of the 16-week internships in internal medicine were arranged for these three systems, so that the interns would have enough time to learn the diagnosis and treatment of the commonly seen diseases. The remaining $1 / 3$ of the time was arranged for the kidney, blood, and endocrine department to enhance the interns' learning of the rarely seen diseases. Through a priority-based approach, the interns would be able to fully grasp the diagnosis and treatment of medical diseases and to distinguish between the diseases, so as to strengthen their capabilities of analyzing and solving problems.

\subsection{Residency and training.}

During the first week of internship, the senior doctors would take four to eight hours to instruct the X-ray features of common diseases in the respiratory and digestive systems, as well as the ECG tracing methods and the ECG changes of common diseases in the circulatory system.

\subsection{Opportunities to independently take care of patients.}

Each intern was asked to take care of two to three ward beds: Check the ward half an hour ahead of schedule, make a doctor's advice according to the conditions of each patient, and implement it with the mentor's approval. In the discussions of complicated and death cases, the interns were allowed to take the floor with sufficient preparation, and their valuable opinions should be confirmed timely. By doing so, the interns would develop a sense of independence and trust, which could fully ignite their enthusiasm for learning and further improve their competence in practices.

\subsection{Guidance of mentors.}

The knowledge gained by the interns comes from both books and experienced mentors, who must be a senior resident doctor or higher-level staff with clinical experiences. They taught the interns the experience and lessons from their own practices through mentoring. Moreover, they should make use of the good virtues, scientific approaches, and pragmatic work ethics to imperceptibly influence and inspire the interns. As a result, through the internship, the interns would learn not only the medical knowledge in clinical practices, but also the characters and integrity of a true doctor. Therefore, the goal of imparting knowledge and cultivating people could be achieved.

\subsection{Training of basic clinical skills.}

To strengthen the interns' capabilities, during the 16-week internship, the interns were thoroughly trained to collect medical history, conduct physical examinations, write medical records, perform cardiopulmonary resuscitation and various punctures, read X-ray films, and trace ECG. These skills were repeatedly emphasized and practiced so that the interns could basically read a plain chest X-ray, correctly trace the ECG, and accurately identify arrhythmia (the features of ECG changes in sinus tachycardia, atrial and ventricular premature beats, atrial fibrillation, and acute myocardial infarction) at the end of the internship. All of these were assessed and recorded as part of the internship results.

\subsection{Regular teaching ward rounds.}

To further improve the interns' comprehensive capabilities to analyze and solve problems in clinical practices, a teaching ward round for typical or complicated cases should be conducted monthly by the department director or senior doctors. An approach with the interns performing and the mentors 
hinting was applied. Collecting medical history, conducting physical examination, making diagnosis, and developing treatment plans were all performed by the interns themselves, while the mentors only helped by offering hints, summaries, and conclusions. The interns were fully mobilized through the ward rounds, and the mentor-intern relationship was more harmonious, creating an active teaching environment.

\subsection{Special lectures.}

To keep the interns updated to the rapid development and latest progress of internal medicine, special lectures should be given on a regular basis by the experts and professors with rich theoretical knowledge and clinical experience. In addition to the key points and difficulties required by the teaching outlines, novel knowledge, innovative methods, and latest progress should also be covered. This would broaden the interns' vision, enrich their clinical experience, and pave a better road for their future learning and professional career.

\section{Innovations}

\subsection{Talent model training.}

Through this series of reform and practices, the hands-on and clinical practice capabilities of graduates of 2011 and 2012 clinical medicine completing internship in our hospital were significantly better than the control group. And they were able to quickly get started as a doctor, which was in striking contrast to the previous interns who were better in theories than in practices. Moreover, it also provided a reference model for the cultivation of interns in the new era.

\subsection{Combination of reform and construction.}

In practice, we adhered to the guiding principle of "promoting construction through reform, while focusing on construction". In the training of the interns, we were able to enrich, refine, and complete various assessment scales and to develop feasible internship plans. While fostering the interns, it also laid the foundation for deepening teaching reforms and strengthening the construction of all disciplines in medicine.

After two years of clinical practice, it was found that enhancing the content, training, and practices of diagnostics teaching in internal medicine internship was greatly helpful in improving the interns' hands-on and clinical capabilities. It has been generally appreciated by the interns and benefited both the mentors and the interns. It has achieved the goals of both accumulating clinical teaching experience and training qualified medical talents.

\section{References}

[1] Zhuanzhen Zheng,et al.Analysis and discussion about reform of internal medicine teaching.Analysis and discussion on the reform of internal science teaching.Basic Medical Education 2016,(18)02,117-120.

[2] Shengtao Li,et al.The exploration and practice of innovative teaching mode of internal medicine in traditional Chinese medicine based on "student-centered" educational concept.Lishizhen Medicine and Materia Medica Research 2016,(27)11,2770-2772.

[3] Yingying Liu Thoughts on the reform of teaching model of internal medicine practice of clinical medicine (general medicine).China Continuing Medical Education 2015,(7)14,3-4.

[4] Jing Wu,et al.Reform and exploration of education of medical diagnosis and internal medicine of eight-year program.Basic Medical Education 2014,(16)07,538-541.

[5] Xing Hao,et al.Analyze the reform of diagnostic teaching from the perspective of clinical internship.Chinese Community Doctors 2017,(33)12,153+155.

[6] Dongwei Zhang,et al.Discussion about exploration and practice of teaching reform of physical 
diagnosis.Health Vocational Education 2016,(34)16,43-44.

[7] Haiyan $\mathrm{Hu}$,et al.Strengthen the reform of diagnostics teaching to enhance the competence of medical students.Journal of Qiqihar University of Medicine 2016,(37)20,2589-2590. 